Service social

\title{
Agression sexuelle et thérapie traditionnelle : essai d'expertise et de prise en charge ethnopsychanalytique d'une adolescente africaine migrante victime d'abus sexuels
}

\section{Louis Serge Aboudé}

Volume 63, numéro 1, 2017

Interventions en matière d'agressions sexuelles

URI : https://id.erudit.org/iderudit/1040029ar

DOI : https://doi.org/10.7202/1040029ar

Aller au sommaire du numéro

Éditeur(s)

École de service social de l’Université Laval

ISSN

1708-1734 (numérique)

Découvrir la revue

Citer cet article

Aboudé, L. S. (2017). Agression sexuelle et thérapie traditionnelle : essai d'expertise et de prise en charge ethnopsychanalytique d'une adolescente africaine migrante victime d'abus sexuels. Service social, 63(1), 43-54. https://doi.org/10.7202/1040029ar
Résumé de l'article

Dans cet article, nous interrogerons en trois étapes certains faits ambigus rencontrés par des professionnels au cours d'une pratique en clinique transculturelle. Le premier pas questionne l'interculturalité des concepts et les réactions du migrant, qui mettraient à mal le professionnel et l'obligeraient à se décentrer, à penser sa position clinique et, enfin, à travailler le contre-transfert culturel négatif qui en résulterait. La deuxième étape concernera l'indication proprement dite du soin dans ce contexte et nous ferons le choix de mobiliser pour ce faire l'ethnopsychanalyse. Enfin, il s'agira de réfléchir aux perspectives cliniques et psychopathologiques qui émergent du matériel clinique récolté à la suite d'une telle rencontre.

Nous poserons ainsi les jalons d'une conceptualisation en psychopathologie de famille démigrante ou remigrante en souffrance, ou encore de patient démigrant ou remigrant, en s'appuyant sur le cas d'une adolescente victime d'abus sexuels. Nous verrons que c'est à l'occasion d'un retour de la famille au Cameroun et de l'intrusion d'une pratique traditionnaliste par la grand-mère (test traditionnel de virginité) qu’un premier événement traumatique vécu en France fera retour après-coup. Cette situation mettrait en lumière de manière particulièrement crue la difficile question du choc toujours possible entre deux cultures thérapeutiques. 


\title{
Agression sexuelle et thérapie traditionnelle : essai d'expertise et de prise en charge ethnopsychanalytique d'une adolescente africaine migrante victime d'abus sexuels
}

\author{
ABOUDÉ, Louis Serge
}

\section{RÉSUMÉ}

Dans cet article, nous interrogerons en trois étapes certains faits ambigus rencontrés par des professionnels au cours d'une pratique en clinique transculturelle. Le premier pas questionne l'interculturalité des concepts et les réactions du migrant, qui mettraient à mal le professionnel et l'obligeraient à se décentrer, à penser sa position clinique et, enfin, à travailler le contre-transfert culturel négatif qui en résulterait. La deuxième étape concernera l'indication proprement dite du soin dans ce contexte et nous ferons le choix de mobiliser pour ce faire l'ethnopsychanalyse. Enfin, il s'agira de réfléchir aux perspectives cliniques et psychopathologiques qui émergent du matériel clinique récolté à la suite d'une telle rencontre.

Nous poserons ainsi les jalons d'une conceptualisation en psychopathologie de famille démigrante ou remigrante en souffrance, ou encore de patient démigrant ou remigrant, en s'appuyant sur le cas d'une adolescente victime d'abus sexuels. Nous verrons que c'est à l'occasion d'un retour de la famille au Cameroun et de l'intrusion d'une pratique traditionnaliste par la grand-mère (test traditionnel de virginité) qu'un premier événement traumatique vécu en France fera retour aprèscoup. Cette situation mettrait en lumière de manière particulièrement crue la difficile question du choc toujours possible entre deux cultures thérapeutiques.

Mots-clés: abus sexuels, clinique transculturelle, interculturalité intersubjective, contre-transfert culturel négatif, approche ethnopsychanalytique, test tradithérapeutique de virginité.

\begin{abstract}
In this article, we will examine in three stages some ambiguous facts encountered by professionals during a practice in a transcultural clinic. The first step concerns the interculturality of the concepts and the reactions of the migrant, which would undermine the professional and oblige him to question himself, to think of his clinical position and finally to work on the resulting negative cultural countertransference. The second stage will concern the actual indication of care in this context and we will choose to mobilize ethnopsychanalysis for this purpose. Finally, it will be necessary to reflect on the clinical and psychopathological perspectives that emerge from the clinical material.
\end{abstract}

We will thus set the stage for a conceptualization in the psychopathology of a demigrant or remigrant family in suffering, or as a demigrant or remigrant patient, based on the case of a teenage victim of sexual abuse. We shall see that it is on the occasion of a return of the family in Cameroon and the intrusion of a traditional practice by the grandmother (traditional test of virginity) that the first traumatic event lived in France will make return after blow. This situation would shed light particularly on the difficult question of the always possible shock between two therapeutic cultures.

Keywords: sexual abuse, transcultural clinic, intersubjective interculturality, negative cultural countertransfer, ethnopsychanalytic approach, virginity tradithérapeutic test. 


\section{INTRODUCTION}

"La clinique a une histoire et une géographie, elle nécessite de prendre en compte les éléments du contexte qui la structurent (...) J'ai développé une clinique transculturelle de ces enfants qui prend en compte leur singularité et leur créativité, pour transformer le risque transculturel lié au fait de grandir dans un autre pays que celui de ses parents, parfois dans une autre langue. Cette situation vulnérabilise les parents, l'enfant et le lien parents-enfants. » (Moro, 2010)

Cette citation de Moro (2010) introduit bien l'objectif de notre réflexion qui se centrera d'abord sur l'interculturalité des concepts et des réactions rencontrés dans la clinique du migrant et qui mettent à mal le praticien africain, notamment pour penser sa position dans la rencontre. Nous pourrions parler d'une situation clinique d'interculturalité intersubjective au cours de laquelle le praticien africain se retrouverait interpellé par des réactions et des concepts difficilement transformables en représentations-choses.

Ensuite, notre réflexion interrogera l'indication du modèle clinique pour penser cette interculturalité intersubjective. À cet effet, le choix d'une clinique transculturelle d'orientation ethnopsychanalytique nous a semblé le plus approprié. Enfin, nous proposerons une réflexion sur l'expertise et les perspectives cliniques du matériel clinique récolté à la suite d'une telle consultation.

Un questionnement nosologique nous paraît d'emblée nécessaire : l'appellation famille migrante en souffrance serait-elle indiquée pour nommer une famille issue d'un pays $X$, ayant migré dans un pays $\mathrm{Y}$, de retour dans le pays $\mathrm{X}$ ? Ou encore l'appelation personne migrante en souffrance, pour un individu né dans un autre pays que celui dont ses parents sont originaires et de retour dans le pays d'origine de ses parents. L'appellera-t-on migrant en souffrance au cours d'une pratique clinique dans ce pays ? Sans toutefois en constituer un point de réflexion à part entière, nous jetterons donc les jalons d'une conceptualisation en psychopathologie et nous proposerons de parler de remigrant ou démigrant en souffrance pour ces situations particulières. Cette proposition est née de ma rencontre clinique avec une adolescente en souffrance, âgée de 16 ans et victime d'abus sexuels, qui consultait à la demande de ses parents. Elle est née en France de parents africains, y a vécu pendant 14 ans sans jamais visiter le pays d'origine de ses parents, pour finalement être confrontée à leur démigration ou remigration au Cameroun deux années avant notre rencontre.

\section{La problématique de l'interculturalité des concepts dans la pratique clinique transculturelle}

Le praticien africain va ainsi devoir travailler un matériau psychique issu de la verbalisation du démigrant ou remigrant en souffrance, qui risque de mettre à mal sa capacité à se représenter les mots de son patient en choses concrètes pour lui. C'est en effet un risque transculturel d'appartenir au même pays d'origine mais d'être né et d'avoir grandi dans un autre pays et dans une autre langue que celle de ses parents et du praticien. Nous irions plus loin et parlerions dans ce cas d'une intrication psychique complexe du praticien, suite à une méconnaissance de la réalité culturelle occidentale contenue dans le discours du démigrant ou remigrant en souffrance. Le risque serait très grand que cette intrication aboutisse à un contre-transfert culturel négatif, aux conséquences fatales pour la clinique.

«La notion de contre-transfert culturel souligne la dimension culturelle donc collective des réactions. Ces réactions de contre-transfert culturel obligatoires et nécessaires ont des 
implications cliniques. II est donc important que ces réactions soient identifiées puis élaborées si l'on ne veut pas faire violence au sujet en le niant dans les différentes composantes de son être et dans son histoire métissée. Pour cela, il est nécessaire d'être capable de se décentrer culturellement, ce qui est loin d'être évident. » (Rouchon, Reyre, Taïeb et Moro, 2009)

La fatalité viendrait du fait que le praticien africain rencontre le démigrant ou remigrant en souffrance dans le même encodage culturel que le sien et se dise: "C'est un Africain en souffrance du même pays et de même culture que moi. "Ce praticien ne tiendrait alors pas compte de l'exil culturel et ne pourrait pas identifier suffisamment les enjeux de l'interculturalité intersubjective dans la rencontre.

Très souvent, ce jeune praticien africain n'a jamais voyagé ni vécu en Occident. La mondialité ne semble même pas l'avoir intégré, malgré les nouvelles technologies de l'information et de la communication dans la plupart des pays d'Afrique. De ce fait, sa connaissance de la culture occidentale risque de n'être que livresque et détachée de la réalité. II n'a presque pas de représentations du métro, du TGV, des trams, des gratte-ciels, des toits végétalisés, des autoroutes, des "voyages pour congé de la Toussaint », et enfin des heures de repas (en Afrique, quand il y a à manger, on mange quelle que soit l'heure). Les réactions de l'altérité démigrante ou remigrante sont parfois prises au piège d'une interprétation conforme au vécu culturel du praticien africain et non à celui de son patient démigrant.

Ces remarques nous amènent à penser qu'il est ainsi nécessaire pour ledit praticien de se déplacer, de se décentrer, de se risquer à l'altérité afin de travailler et de penser cette interculturalité intersubjective au cours de la rencontre clinique. II sera également crucial de mobiliser un modèle clinique qui prend en compte cette interculturalité intersubjective, d'où notre option en faveur de l'ethnopsychanalyse.

\section{Une interculturalité intersubjective à penser, dans une clinique interculturelle d'orientation complémentariste du migrant : la clinique d'Aïcha}

\section{Aïcha et ses liens familiaux}

Aïcha est une adolescente de 16 ans, aînée d'une fratrie de trois enfants ( 2 garçons et elle), et de parents musulmans du nord du Cameroun. Je la rencontre en consultation, après une campagne de sensibilisation dans le cadre d'un projet de "lutte contre les agressions sexuelles, les discriminations sexistes et l'exploitation sexuelle des enfants " d'une ONG nommée le CIPCRE Cameroun ${ }^{2}$. Son tableau symptômatologique d'automutilation, d'inadaptation sociale, de fugues, d'échecs scolaires, de phobies, de cauchemars et d'insomnies a mené ses parents à me solliciter pour une consultation psychologique.

Elle est née en France, de parents camerounais. Elle y a vécu pendant quatorze ans, sans jamais rentrer au Cameroun. Ses parents et tous les enfants sont rentrés depuis deux ans, suite à une nouvelle affectation au Cameroun. La fonction des parents les contraint à voyager régulièrement hors du pays. Suite à cette absence, l'autorité parentale est assurée, de plein droit, par Mamie, la grand-mère paternelle d'Aïcha qui habite le domicile de son fils Moussa, le papa d'Aïcha. Mamie

\footnotetext{
1 Une avancée nécessaire pour définir la mondialité comme parade à la délétère mondialisation (le Tout-monde et le Tout-empire). Aussi fertile qu'ambitieuse, l'écriture d'Édouard Glissant a choisi pour objet la dynamique accélérée d'un monde en voie de créolisation généralisée : le Tout-monde (Glissant, 2005).

2 CIPCRE Cameroun est une ONG qui travaille dans le domaine social et précisément pour la lutte contre les abus sexuels, les discriminations sexistes et l'exploitation sexuelle des enfants, ainsi que le veuvage des femmes.
} 
jouerait donc le rôle de Surmoi. Aïcha et ses frères ne parlent pas et ne comprennent pas le fufuldé ${ }^{3}$, la langue maternelle de leurs parents. La grand-mère ne parle pas et ne comprend pas le français. Aucun enfant n'avait connu le Cameroun, ni la grande famille, et encore moins leur grand-mère, sinon en photo. Les liens entre les enfants et la famille se sont en quelques rares fois noués par téléphone. À la différence des liens resserrés entre les enfants, qui s'entendent bien, règnent essentiellement un conflit et une rivalité entre Aïcha et sa grand-mère. Une rivalité et un conflit créés par l'autorité exacerbée, l'intrusion intempestive, le perfectionnisme, le traditionnalisme et le fanatisme musulman de Mamie, dixit Aïcha.

Face à cette catégorie de famille démigrante ou remigrante en souffrance, dont Aïcha serait l'enfant symptôme, notre approche clinique se fonderait-elle seulement sur la psychanalyse ? Et dans ce cas, comment justifier l'utilisation d'une telle approche, au regard des chavirements cliniques psychanalytiques rencontrés dans de pareils cas ? Sinon, comment donc répondre à ces tensions psychiques imbriquées par des mélanges culturels du démigrant ou remigrant en souffrance, après plusieures années d'acculturation, qui déboucheraient à la fin, sur une interculturalité intersubjective des concepts et des processus psychiques complexes et difficiles à penser.

C'est ce qui explique, en tout état de cause, notre choix d'une pratique clinique où, au moins, les approches psychanalytique et transculturelle seront associées dans une perspective devereusienne, et donc complémentariste. En d'autres termes, une pratique clinique ethnopsychanalytique qui se base sur le complémentarisme.

C'est une méthode qui prévoit l'utilisation "d'une pluridisciplinarité non fusionnante et non simultanée, celle du double discours obligatoire... où toutes ces approches sont utilisées de manière obligatoire mais non simultanée» (Devereux, 1972), "pour permettre une lecture plus fine et plus complète du corpus clinique d'une psychothérapie transculturelle » (Moro, 2010).

Les disciplines à la base de cette méthode que nous utilisons sont l'anthropologie, pour décoder les sens collectifs, le contenant, et la psychanalyse, pour comprendre les mouvements intrapsychiques, le contenu. Nous nous sommes décentrés quelques fois pour travailler le niveau culturel, le niveau individuel et leur interaction.

La transculturalité dont nous parlons ici se justifie du fait que nos entretiens avec Aïcha se sont déroulés en français, et en fufuldé pour sa grand-mère qui l'encadre depuis son retour au Cameroun. Pour l'entretien avec Mamie, ses propos m'étaient directement traduits en français et mes interventions traduites en fufuldé.

\section{Le dispositif clinique}

Au premier entretien, je suis assis en face d'Aïcha et de ses parents. Elle est assise entre ses deux parents et une table nous sépare. La stagiaire est assise à côté de moi et en face des trois : un dispositif aux insuffisances avérées mais en Afrique, on travaille avec les moyens du bord. Au moins, nous nous réjouissons d'être tous assis au cours de cette séance. La suite des entretiens se fera en l'absence des parents, suite à leur indisponibilité. La grand-mère paternelle d'Aïcha, qui ne parle pas et ne comprend pas le français, prendra le relais. Je ne comprends pas le fufuldé, encore moins l'encodage culturel musulman et traditionnel de cette région du Cameroun.

Pour transcrire et comprendre au plus fin près ce qui se dit dans chaque consultation, je me fais donc aider d'une stagiaire en psychologie. Elle est de la même culture régionale que la famille

\footnotetext{
${ }^{3}$ Le fufuldé est une langue bantoue du nord du Cameroun.
} 
Moussa. Elle parle très bien cette langue et en comprend bien les références culturelles et religieuses. Elle joue donc un triple rôle : celui de transcriptrice, d'interprète et de médiatrice culturelle. Je suis bien conscient de quelques problèmes liés à la transcription, qui pourrait être biaisée par la perception ou la compréhension qu'en a ma transcriptrice, mais cela me semble la meilleure alternative.

Au cours des entretiens où la grand-mère intervient, je m'adresse à la stagiaire qui traduit mes propos, et vice versa. Au début de la séance, je me présente et présente la stagiaire, puis j'énonce les motifs et les objectifs de notre rencontre, qui peuvent se complexifier au fil des entretiens et des différents discours recueillis. Nos séances durent une heure et demie et ont lieu chaque mardi, à la même heure. Ce rendez-vous est arrêté d'un commun accord avec Aïcha. J'occupe le rôle du clinicien principal au cours de toutes les rencontres.

\section{La première rencontre avec Aïcha : la verbalisation refoulée ou bien l'inhibition occasionnée?}

Cet entretien se déroule en présence de ses deux parents qui l'accompagnent ce jour-là. Avec sourire, elle accepte de répondre à mon entretien d'identification, de préparation, de mise à l'aise et d'évaluation psychologique. D'entrée de jeu, je rencontre une adolescente calme, au large sourire et ouverte à moi, même si son élocution est quelque fois entrecoupée par le mordillement de ses lèvres. Ses yeux sont noirs, grand ouverts comme pour bien apercevoir ce qu'elle n'arrive pas à bien percevoir. Je découvre aussi qu'elle a l'air hagard, puisqu'elle observe la salle d'écoute comme pour retrouver quelque chose et marquer de sa présence l'espace qui s'impose à elle. Mais ce regard devient subitement fuyant lorsqu'il rencontre le mien et s'abaisse rapidement. Estce pour refuser l'alliance clinique intersubjective que j'essaye de tisser avec elle ? Ou sinon, se questionne-t-elle sur sa raison d'être dans cette salle? Ensuite, elle feint de dégager une bonne humeur et un bien-être, qui s'éclipsent très rapidement lorsque j'évoque les motifs et le but de notre rencontre: notamment, cette souffrance enkystée dans sa psyché, dont j'ai rappelé la symptomatologie plus haut. À la seconde, je ressens avoir créé en elle, un dégoût et une haine pour ma personne. Son visage se referme et elle me fusille du regard. C'est un transfert négatif que je travaillerai. Mais j'essaye, avec l'aide de ses parents qui lui demandent de ne pas être vilaine à mon égard, d'établir une relation stable afin de retisser notre alliance. Avec sourire, convivialité et empathie, je lui reprécise mon rôle au cours de la consultation, mon estime pour elle, et ma volonté de l'aider à penser sa souffrance. C'est un contre-transfert affectif que je travaille. Malheureusement, elle refuse de parler car elle n'a pas envie de parler. Je récolterai l'anamnèse de sa souffrance à la suite de l'entretien avec ses parents.

À la fin de cet entretien, je me pose la question de savoir si, la présence des parents n'inhiberait pas sa verbalisation?

Deuxième rencontre avec Aïcha et Mamie : de l'inhibition à la verbalisation, et de la verbalisation aux catastrophes de symbolisation

La question précédente semble bien posée, car au cours de cet entretien où Mamie l'accompagne, Aïcha verbalise avec spontanéité et fluidité. Elle se plaint de l'autorité exacerbée, de la privation de toutes ses libertés et du fanatisme musulman de sa grand-mère, ce qui l'énerve, et elle y répondrait par son autoflagellation, son automutilation et son isolement. Elle me confie : " Beh... Il y a beaucoup de choses qui m'énervent ... Je suis triste, monsieur... Mamie me fait beaucoup de mauvaises choses... chais pas... ( silence et regard à sa grand-mère), elle me colle au cul... Quand Mamie me fait ses choses-là, je me sens très mal... Ca m'énerve... ça m'énerve... ça m'énerve et... ça... me rappelle des choses, purée !... des choses qui se sont faites quand 
j'avais 8 ou 10 ans... Et quand je me cache seule dans ma chambre... heu... je me giffle, ou je me blesse... (silence+++) là je me sens un peu bien... "Aïcha bouge ses jambes et regarde sa Mamie, qui ne comprend rien de ce qu'elle dit, et qui cherche d'ailleurs à le comprendre. J'interdis à la médiatrice de traduire en fufuldé. Et après un silence relativement long, Aïcha : «Behhh... même s'il ya eu certaines choses, beh je suis sûre et certaine d'être encore vierge... hep »

Elle ouvre grand les yeux, me regarde, puis regarde à nouveau sa grand-mère. Ensuite, suivra un silence total, et pas un mot pendant trois à cinq minutes. J'observe juste à travers ses yeux grand ouverts que son regard évoquerait une surprise. Dans sa verbalisation, aurait-elle dévoilé un secret longtemps enfoui dans son jardin secret ? Peut-être voulait-elle uniquement se plaindre des vicissitudes de sa grand-mère qui l'énervent, cette grand-mère intrusive dans sa vie ? Mais auraitelle, par inadvertance, dévoilé une sexualité certes précocement vécue, mais non traumatique à cette période-là?

J'essaye de marcher avec elle, dans ses catastrophes de symbolisation qui viennent de remplacer sa verbalisation. II s'agit d'" une mise à défaut du travail psychique fondateur, une mise en défaut du lien entre représentation-chose et représentation-mots (...) Mais elles expriment aussi, l'espace d'une réorganisation sous le primat du somatique »(Roman, 1997).

D’abord les bras croisés, puis décroisés, ensuite ses mains levées vers le ciel et enfin relâchées. Tantôt debout, puis assise, elle ne parle plus, sinon uniquement avec son corps. Elle est soudainement prise d'une transpiration corporelle et d'une angoisse souterraine. Je puis ressentir, à un mètre de distance, les vibrations émises par les puissants battements de son cœur. Cette inhibition verbale et cette sémiologie corporelle n'exprimeraient-elles pas une angoisse liée à une extrême souffrance vécue ? Une souffrance dont elle se serait retirée par un déni, jusqu'à ce qu'elle chavire après la verbalisation «Behhh... même s'il y a eu certaines choses, beh je suis sûre et certaine d'être encore vierge... hep... purée? »

Je m'appuierai sur ma médiatrice culturelle, pour en savoir plus sur les imbrications anthropologiques de la perte de virginité dans la tradition musulmane et du nord du Cameroun dont Aïcha fait partie. Cela me semble porteur de sens pour la suite de la clinique transculturelle. Je n'abandonnerai donc pas cela comme un fait banal, j'y travaillerai par la suite.

Après quelques minutes de ces catastrophes de symbolisation, elle renoue avec la parole, comme résignée d'avoir déjà dit ce qu'il ne fallait pas dire. Elle poursuit sa verbalisation en me confiant : "Behh ce n'était que des jeux... En France, je faisais des choses avec des garçons, ça m'amusait... (rires) Je n'étais pas en colère... Je me masturbais en France et même mes agressions sexuelles ne me posaient pas de problèmes d'insomnie, de cauchemars. Mais depuis que je suis rentrée au Cameroun et que Mamie me traumatise avec ses histoires de test pour savoir si je suis encore vierge, j'ai commencé à avoir honte de moi et à me rendre responsable de tout cela. Mais avant, c'était un jeu, même s'il était interdit... Mais les autres choses n'étaient pas des jeux... (Silence +++). Quand j'avais heu... 8, non heu 9 ou 10 ans, j'ai été violée plusieurs fois par un voisin, le papa de notre amie X. Behh, je ne l'ai jamais dit à personne et je ne me suis jamais sentie mal. (Silence+ et regarde sa mamie qui nous regarde avec des yeux grand ouverts). C'est depuis que je suis rentrée ici et que Mamie me fait des mauvaises choses... heu que je me sens mal... Elle contrôle mon habillement, m'empêche de sortir seule... elle me colle au cul... purée...elle est présente partout, dans la cuisine, au salon, dans ma chambre, elle me fait des choses pour savoir si je suis toujours vierge, et me dit que je suis une infidèle de l'islam, je serai maudite... Beh c'est là que j'ai commencé à avoir honte de moi, à avoir peur et à vouloir rester seule. Je me culpabilise que je ne devrais peut-être pas le faire. Elle me fait boire des écorces pour me soigner de tout cela. Je ne veux même plus boire ça... » 
Cette verbalisation n'évoquerait-elle pas une tentative d'élaboration traumatique, une acceptation de la perte d'objet (la virginité) et un début de subjectivation chez cette démigrante en souffrance ?

À la suite de l'entretien, Aïcha continue de verbaliser sa souffrance en me confiant que, le comportement de sa grand-mère serait à l'origine de toute sa souffrance, y compris ses phobies. Selon elle, ces phobies se nourriraient aussi de la précarité et de l'insécurité de son nouvel environnement de vie. "Beh ici il ya trop d'obscurité dans la ville, dans les maisons, dans les rues... et ça me fait peur... (elle accompagnane sa verbalisation d'un geste des mains)... beaucoup d'obscurité dans la ville... pas de métro... pas de bus et pas de tram. Je ne dors pas la nuit parce que j'ai peur... j'ai aussi des cauchemars et beaucoup d'insomnie! (elle tourne son regard vers la porte)... Dans la nuit, je me fais des films par rapport à ce que Mamie me dit... me fais... ici on vit comme en prison! Pas de voyages d'études à l'école, pas de congés de la Toussaint, pas de parc et donc pas de divertissements... Mais putain... Purée rien que me coller au cul (elle regarde sa mamie qui n'hésite pas à lui demander ce qu'elle raconte. Aucun message n'étant décodé, je lui demande de poursuivre sa verbalisation). Je n'ai pas envie d'aller à l'école sans métro, sans bus et sans tram. Et quand ca me fait mal... j'ai mal, je me gifle... je me saigne... et ça passe. (Avec un geste ferme des deux mains) Et d'ailleurs, elle n'aura qu'à aller se faire foutre dorénavant. »

Je suis soudainement pris d'un malaise contre-transférentiel culturel dont je parlais au début de ma réflexion. En tant que praticien africain qui n'a pas encore voyagé en Occident, mon malaise est celui de me retrouver interpellé par des mots difficilement transformables en représentationschoses, et une gestuelle que je nommerais " agressivité et mauvaise éducation ». Ce qui ne serait pas forcément perçu ainsi dans la tradition occidentale où a vécu Aïcha. Mon malaise est de ne pouvoir me déplacer et me décentrer, face à cette interculturalité de concepts et de gestes qui me perdent.

Malgré mon malaise, je l'aide à verbaliser ses phobies, pour lui permettre d'identifier ses frayeurs. J'établis donc une nouvelle alliance qui, en même temps, lui permettrait de les élaborer et de les maîtriser. Je constate cependant qu'elle essaye de me substituer à son papa, toujours absent lorsqu'elle a besoin de lui. "Vous voyez monsieur, mon papa n'est jamais là lorsque j'ai besoin de lui. Sa femme et lui sont toujours partis... chais pas pourquoi ? Vous au moins êtes là... Heu gentil... je me sens... heu... beh... comme... Je suis votre fille... (elle sourit) avez-vous une fille comme moi ? elle me ressemble?»

C'est un transfert positif que je travaille. II sera porteur d'une nouvelle dynamique, qui lui permettrait de travailler la carence affective parentale, et l'aiderait même à désinvestir ce conflit généralisé. Peut-être aimerait-elle se voir aimée et protégée comme un enfant. Mais ses parents ne sont jamais présents pour lui offrir des bras rassurants lorsqu'elle en a besoin. Son clivage confusionnel qui l'amène à appeler sa maman "la femme de mon père » renforcerait notre hypothèse d'une carence affective avérée.

À la fin de cet entretien, il me semble clairement indiqué que la toile de fond clinique transculturelle d'orientation ethnopsychanalytique, pour une prise en charge singulière et collective de la famille est nécessaire. L'objectif sera d'essayer, bien qu'en l'absence des parents qui ne peuvent malheureusement pas être disponibles, de reconstituer une enveloppe culturelle et psychique de la famille. Pour compléter les éléments de cette toile de fond, j'invite cette fois-ci Mamie à la prochaine consultation. D'ailleurs comment pourrait-il en être autrement puisque c'est de par son autorité qu'elle aurait réveillé ce brasier familial, camouflé par une épaisse couche de cendre de paix. 
Troisième rencontre avec Mamie : une verbalisation qui appelle une lecture anthropologique

Au cours de cet entretien avec Mamie et Aïcha, le dispositif thérapeutique décrit plus haut reste le même. Mais à cette occasion, je m'adresse en français à la médiatrice, qui fait la traduction en fufuldé à la grand-mère et me retraduit ses propos. Je n'aborde pas d'entrée de jeu, la question des abus sexuels d'Aïcha, mais plutôt son comportement inadapté qui poserait problème. À peine a-t-elle reçu la traduction de mon propos, qu'elle déclenche une logorrhée verbale chronique. Je m'y abreuve par le truchement de ma stagiaire. Mais je me rends très vite compte de ses difficultés à traduire, à transcrire et à nous écouter en même temps. Je commence également à prendre certaines notes de notre entretien, traduits en français. Mamie me confie : "Ma fille Aïcha est une fille ordinaire qui développe des comportements extraordinaires... mon fils, cette fille a des comportements très étranges... kaï... kaï... yééééé, (s'écrit-t-elle avec émotion en levant les mains vers le ciel)... Mon fils... (en me touchant et en me tapotant...) je pense d'ailleurs qu'elle est habitée par le démon. Depuis son retour au Cameroun, elle ne développe que des comportements dignes d'une infidèle ${ }^{4}$. Que ce soit dans son vestimentaire, dans sa manière d'être ou de faire, dans ses propos, son vécu relationnel avec les autres, ma petite-fille montre qu'elle est une infidèle... kaï... Yééééé qu'Allah fasse quelque chose... c'est l'école des Blancs qui a gâté l'éducation de ma petite. Regarde la qualité d'habit qu'elle voudrait porter... Des vêtements que des jeunes filles gâtées pourries portent ici dehors. Dans sa garde-robe venue de France, je n'ai trouvé aucun vêtement digne de la tradition islamique; uniquement des habits que les jeunes gâtés portent... qu'elle appellent si c'est quoi là, ohhh ? ou bien les DVD, des VCD et des $P D D^{5}$ oh ? Mon fils, dis-moi toi-même comment la religion islamique s'épanouirait-elle avec une telle éducation des Blancs ? Avec des comportements comme ça... kaï ? (Elle tire l'oreille d'Aïcha, la pousse de sa chaise et me pointe du doigt.) C'est votre école-là... des Blancs qui accorde trop de libertés aux jeunes filles en particulier, et aux femmes en général. Dis-moi, ma fille, (elle s'adresse à la médiatrice), comment comprendre qu'une fille de cet âge ose demander la permission de sortir faire des loisirs, même si c'est en journée ? comment des filles de cet âge peuvent-elles regarder des émissions de télévision où des hommes et femmes se touchent bizarrement? D'ailleurs comment une fille de cet âge n'a pas encore été envoyée en mariage ? Elle est déjà gâtée... » (Silence, elle secoue la tête en signe de négation.)

À la suite de l'entretien, je sens monter sa détonation verbale et ses dents se resserrer. Son ton très autoritaire m'explique les sacro-saints principes musulmans, qui devraient régir le vécu de l'homme. J'ai l'impression de prendre part à un cours sur l'Islam. Au cours de cette détonation verbale, un élément se glisse dans sa verbalisation.

«Mon fils... (presque en larmes)... J'étais très choquée et déçue lorsque cette fille est arrivée ici au Cameroun... hum ! Je lui ai fait un test traditionnel de virginité6. Imagine ce que j'ai découvert ? la honte, le déshoneur de toute la famille... kaï... Ma fille avait déjà été déshonorée, elle n'était plus vierge. Heureusement que je l'ai gardé en secret, sinon la honte pour mon enfant... Heureusement que je vais guérir ça... c'est même déjà guéri... Je lui fais des potions traditionnelles pour reconstituer son hymen. Et ça marche... (Silence +++ , puis elle regarde à

\footnotetext{
${ }^{4}$ L'Infidèle, selon l'islam, est le nom donné à celui qui ne croit et ne pratique pas la volonté d'Allah. Selon la sourate 98 , verset 6 et 7 : «Les infidèles parmi les gens du livre, ainsi que les associateurs, iront au feu de l'enfer, pour y demeurer éternellement. »

${ }^{5}$ Appelation du mode vestimentaire camerounais. DVD désigne, le vêtement dont le « Dos et Ventre sont Dehors », VCD signifie « Ventre et Cuisses Dehors » et enfin PDD signifie « Poitrine et Dos Dehors ».

${ }^{6}$ Le Test de virginité est un test pratiqué dans certaines traditions africaines, qui consiste à faire bouillir un œuf, à le laisser refroidir et à essayer de l'introduire dans le vagin de la jeune fille, afin de s'assurer que l'hymen est en place. Le passage de l'œuf justifie la perte de l'hymen, et donc le déshonneur pour la fille et sa famille.
} 
gauche et à droite.) Je vous supplie d'ailleurs de ne jamais en parler à personne, même pas à mon fils, son papa. »

Après cette révélation involontaire sur le "mariage précoce» et les pratiques thérapeutiques traditionnelles de test de virginité sur sa fille, elle se rétracte et essaye de se jouer de mon intelligence. Avec un sourire malicieux, elle m'affirme et veut me convaincre que sa fille n'a jamais connu d'homme, déniant ainsi la sexualité précocement vécue par sa fille.

«(Sourire) Heureusement que... je sais que... ma fille ne connaît pas d'homme. Son hymen a cédé à cause de l'usage abusif des mauvais produits de toilettes intimes des Blancs... C'est pourquoi... J'en ai d'ailleurs jeté à son arrivée au Cameroun... hihihihihi d'ailleurs ma fille et moi nous entendons très bien... il n'y a même pas de problème... hihihihihi. »

Derrière ce sourire trompeur, se cache un discours recouvert d'un vernis de déni. Elle ignore en effet que sa fille m'a dévoilé avoir été victime d'abus sexuels à l'âge de 8-9 ans. Est-ce pour atténuer le poids symbolique de sa révélation ? En plus, elle qui auparavant me confiait que sa fille se comporte comme une infidèle et qu'elle la réprimande avec violence, me dit entretenir des relations pacifiques avec ses trois enfants. Je l'accepte, mais je pense que règne dans cette famille une paix de cimetières7 qui donne à comprendre le vécu d'un conflit latent entre les deux. Je termine cet entretien en invitant Mamie à me laisser seul avec Aïcha, afin de lui faire passer des tests projectifs. Je les utilise comme support de ma démarche clinique et de la collecte des données pour mes recherches ultérieures. Elle le refusera comme par le passé, et acceptera de me la laisser en compagnie de la médiatrice. Je lui passerai donc les tests projectifs de Rorschach et de TAT en présence de ma médiatrice. Mais dans mon présent propos, je ferai volontairement abstraction des protocoles projectifs, même s'ils ne sont pas moins révélateurs d'un traumatisme sérieusement enkysté. Plus tard, je sollicite également la présence des parents, pour une prise en charge holistique et ethnopsychanalytique familiale. En leur absence, j'essaye de l'effectuer avec les membres de la famille disponibles, au point de ne faire que du surplace après plusieurs mois de clinique. En l'absence d'un cadre juridique assez fort, qui protège aussi bien le soignant que l'enfant, mes efforts à penser cette souffrance individuelle et collective seront obérés.

Mais avant d'en arriver là, j'essayerai d'effectuer un décodage anthropologique sur la perte de la virginité, le rôle de la grand-mère et le mariage des jeunes filles, dans la tradition ethnique en question.

\section{Perte de la virginité, rôle de la grand-mère et mariage précoce : lecture anthropologique pour un essai d'expertise ethnopsychanalytique}

Aidé par ma médiatrice, j'essaye de décoder le sens collectif des éléments cités ci-dessus afin d'étayer mon approche clinique transculturelle. Elle me donne à comprendre que, de manière générale, la perte de la virginité est une étape importante dans le vécu d'une fille. Dans le contexte occidental et sur le plan juridique, la majorité sexuelle des filles serait fixée à 18 ans. La perte de la virginité serait donc admise à cet âge-là, même si à l'épreuve des faits, la précocité sexuelle des jeunes filles n'émeut plus, tant qu'elle ne pose aucun problème physique et psychopathologique. Cependant, le contexte traditionnel africain est très différent, et musulman encore plus. En effet, chez certains groupes ethniques, tout dépendrait de la croissance de la jeune fille et d'avec qui cela se produit. Par exemple, la poussée des seins et l'apparition des premières règles

\footnotetext{
7 J'utilise l'expression «paix des cimetières » ici, pour traduire une paix de façade. En Afrique, on dit des gens des cimetières qu'ils accueillent toutes les dépouilles, y compris celle dont la joie d'y être, n'est forcément pas. Cependant, aucun mort ne contesterait un cimetière.
} 
annonceraient la maturité sexuelle. Dans la tradition musulmane, la perte de la virginité ne serait admise sous aucune autre condition que celle du mariage et après l'apparition des premières règles. De ce fait, toute autre alternative de perte de virginité serait un déshonneur et une honte pour la fille et sa famille.

Comment Aïcha aurait-elle vécu cette perte d'objet, associée à son angoisse de démigration ou remigration au Cameroun? Telle un navire en pleine mer ayant perdu ses amarres, elle serait ballotée entre tempêtes pulsionnelles et calme plat, entre colère et passivité. Ce qui l'amènerait à redéfinir les frontières entre son moi et l'extérieur. En l'absence de référents stables du fait de son errance culturelle et identitaire, elle ne saurait plus sur quel levier s'appuyer. Elle aurait perdu les libertés d'évasion, de divertissement, d'épanouissement, octroyées par l'Occident pendant quatorze années, jusqu'à sa démigration ou remigration deux ans aupravant. Elle serait «mal outillée en face d'un univers culturel dont (elle) ne comprend ni les représentations, ni les valeurs, piégée par le clivage procédant du traumatisme (démigratoire), et parfois même prise dans différents conflits entre les logiques culturelles du pays d'où on vient (la France) et celle du pays d'accueil (le Cameroun), le sujet doit inventer des façons de faire pour ne pas décompenser, pour ne pas s'effondrer, ou pour ne pas se morceler » (Di et Moro, 2008).

Ma médiatrice culturelle m'aide également à décoder le sens du mariage dans ce contexte traditionel. Certains appelleront cela mariage précoce mais je choisis de ne pas prendre part à ce débat. Dans cette tradition, la jeune fille est obligatoirement mariée entre 8 et 16 ans pour préserver sa dignité, sa virginité et, surtout, pour recevoir l'éducation de son époux qui, pour la plupart du temps, est un homme très mature. II peut être de 3 à 5 fois plus âgé que son épouse, qui se compte parmi plusieurs coépouses. J'essaye aussi de comprendre pourquoi la femme est autant protégée, au point d'être enfermée dans un voile ? J'obtiens le même décodage que dans le cas de la perte de la virginité. De ce fait, il y aurait même des alliances de mariage entre des "bonnes familles ", avec la possibilité pour un homme d'y choisir une épouse dès sa naissance. Pour tout accompagnement d'une jeune fille à des fins nuptiales, les grands-mères y ont des places singulières, au sens de la référence traditionnelle et religieuse. C'est elles qui sont censées protéger la jeune fille de l'impureté et des convoitises sexuelles. Elles moulent son éducation aux valeurs traditionnelles jusqu'au mariage. Elles l'accompagnent à passer de fille à épouse, en passant par l'âge de la puberté. Malheureusement, à la petite enfance d'Aïcha, Mamie n'était pas là, et encore moins à sa transition pubertaire. Cette absence/carence aurait-elle pris un coup traumatique à la découverte de la perte de l'hymen de sa fille, au point de conflictualiser leur vécu intersubjectif? Tout en enrichissant ma posture clinique complémentariste, je n'occulte aucunement que les tiraillements entre cultures occidentale et traditionnelle auraient aussi un impact sur la souffrance psychique d'Aïcha et de Mamie. Mamie occupe en fin de compte, une fonction surmoïque archaïque dans cette famille. En d'autres termes, c'est elle qui constituerait l'objet dangereux, de frustrations, de déplaisir, de douleur et de persécution dans cette famille. Ce qui nourrirait donc l'errance entre les deux cultures conflictuelles d'Aïcha, qui oscillerait entre la culture occidentale qui a moulé sa personnalité depuis quatorze ans et la culture traditionnelle qui conditionne son vécu actuel, depuis sa démigration. Elle doit remodeler son moi présent, avec des troubles entre évidences culturelles et évidences naturelles. Elle est passée de la liberté de choisir ses vêtements à l'obligation de porter des vêtements choisis, de la liberté de sortir se divertir à l'enfermement, du dialogue à la crispation, et peut-être même de la liberté sexuelle à la censure de la simple pensée sexuelle. Une liberté sexuelle qu'elle n'aurait pas vécue comme traumatogène par le passé et qui nous ramènerait à la conception freudienne du traumatisme psychique, lorsqu'il évoque la notion d'après-coup (Freud, 1895). 
L'analyse montre qu'il y aurait eu deux temps du traumatisme : en premier lieu, les masturbations et les abus sexuels subis en France, qui ne lui posaient d'ailleurs pas de problèmes psychopathologiques de cauchemars, d'insomnie, de honte et de culpabilité. En deuxième lieu, outre l'inadaptation à son nouvel environnement de vie, le harcèlement thérapeutique traditionnel et l'ingérence de sa Mamie qui, en plus de constituer un nouveau traumatisme aurait déclenché une décompensation. C'est l'événement actuel qui réinvestirait des traces mnésiques d'un événement à caractère sexuel, mais plus ancien et non vécu comme traumatique au moment de sa production. Le deuxième temps du traumatisme et le nouveau traumatisme déboucheraient sur un certain clivage qui amènerait Aïcha à ne pas aimer la partie d'elle qui a vécu en France, même si elle déteste la partie d'elle qui vit des contraintes culturelle actuelles.

\section{Conclusion}

Pour faire face au risque d'un contre-transfert culturel négatif induit par l'interculturalité des concepts et des réactions du démigrant, nous voyons se confirmer la nécessité d'une décentration et d'une ouverture à la mondialité. Quant à l'indication d'un modèle clinique transculturel, le choix d'une seule approche pourrait engendrer des chavirements cliniques qui ouvriraient davantage la porte déjà grand ouverte en Afrique au charlatanisme, par exemple lorsque le sida est encore attribué à la sorcellerie et la souffrance psychique au déferlement des démons. À ce titre, nous pensons être confirmé dans notre choix d'une clinique d'orientation ethnopsychanalytique complémentariste, en ce qu'elle mettrait le bien-être de la personne au centre de ses préoccupations tout en articulant de manière rigoureuse le vécu intrapsychique et le contexte sociohistorique. La notion de l'ambivalence des enveloppes culturelles serait également une ouverture à des débats plus larges en clinique transculturelle.

Louis Serge Aboudé

Psychologue clinicien Université de Lausanne, Suisse

\section{BIBLIOGRAPHIE}

Ausloos, G. (1990). Approche systémique et langages familiaux, Ottawa, Vermette.

Bouaziz, Nora (2013). « Comment accueillir le délire d'un adolescent étranger isolé en consultation transculturelle? ", Adolescence, tome 31, n 3 .

Devereux, G. (1972). Ethnopsychanalyse complémentariste, Paris, Flammarion.

Di, C., et M. R. Moro (2008). «La protection de l'enfance. Entre l'offre institutionnelle et la demande des familles en situation migratoire ", Le Journal des psychologues, vol. 256, $n^{\circ} 3$.

Ey, H., P. Bernard et C. Brisset (1989). Manuel de psychiatrie, Paris, Masson.

Ferenczi, S. (1932). Confusion de langue entre les adultes et l'enfant, Paris, Payot, coll. « Petite bibliothèque Payot » (2004).

Freud, S. (1895). Études sur l'hystérie, Paris, Payot. 
Gabel, M., S. Lebovici et Ph. Mazet (1995). Le traumatisme de l'inceste, Presses universitaires de France.

Glissant, É. (2005). La cohée du lamentin. Poétique V, Paris, Gallimard.

Haesevoets, Y.-H. (2015). L'enfant victime d'inceste. De la séduction traumatique à la violence sexuelle, $2^{\mathrm{e}}$ éd., Bruxelles, De Boeck.

Lagache, D. (1949). L'unité de la psychologie, Paris, Presses universitaires de France.

Lagache, D. (1999). La psychanalyse, Paris, Presses universitaires de France, coll. " Que saisje ? ", $19^{\mathrm{e}}$ éd.

Laplanche, J., J.-B. Pontalis et D. Lagache (1967). Vocabulaire de la psychanalyse, Paris, Presses universitaires de France.

Moro, M. R. (2010). Nos enfants demain, pour une société multiculturelle, Paris, Odile Jacob.

Moro, M. R. (2016). La violence envers les enfants, approche transculturelle, Paris, Fabert.

Roman, P. (1997). "Troubles somatiques et catastrophes de symbolisation », Psychologie clinique et projective, vol. 3.

Rouchon, J.-F., A, Reyre, O. Taïeb et M. R. Moro (2009), "L'utilisation de la notion de contretransfert culturel en clinique », L'Autre, cliniques, cultures et sociétés, vol. 10, n 1.

Tsala Tsala, J. P. (2002). "Adolescence et crise familial en Afrique. Approche systémique d'un cas dans une famille camerounaise ", in J. P. Tsala Tsala (dir.), Santé mentale, psychothérapie et cultures, Vienne, World Council for Psychotherapy. 